\title{
SCALE RESOLVED SIMULATIONS OF THE OECD/NEA-VATTENFALL T-JUNCTION BENCHMARK
}

\author{
Thomas Höhne \\ Institute of Safety Research, Helmholtz-Zentrum Dresden-Rossendorf e.V. (HZDR) \\ P.O. Box 510 119, D-01314 Dresden, Germany, E-mail of corresponding author:: t.hoehne@hzdr.de
}

\begin{abstract}
Mixing of fluids in T-junction geometries is of significant interest for nuclear safety research. The most prominent example is the thermal striping phenomena in piping T-junctions, where hot and cold streams join and turbulently mix, however not completely or not immediately at the T-junction. This results in significant temperature fluctuations near the piping wall, either at the side of the secondary pipe branch or at the opposite side of the main branch pipe. The wall temperature fluctuation can cause cyclical thermal stresses and subsequently fatigue cracking of the wall.

Thermal mixing in a T-junction has been studied for validation of CFD-calculations. A T-junction thermal mixing test was carried out at the Älvkarleby Laboratory of Vattenfall Research and Development (VRD) in Sweden. Data from this test have been reserved specifically for a OECD CFD benchmark exercise. The computational results show that RANS fail to predict a realistic mixing between the fluids. The results were significantly better with scale-resolving methods such as LES, showing fairly good predictions of the velocity field and mean temperatures. The calculation predicts also similar fluctuations and frequencies observed in the model test.
\end{abstract}

\section{Indroduction}

Temperature fluctuations in piping systems can lead to thermal fatigue in the pipe structures. There are examples from nuclear power plants (NPP) where thermal fatigue has been the cause for leaking pipes. When hot and cold fluid join and turbulently mix, often not completely or not immediately, it results in significant temperature fluctuations near the piping wall, either at the side of the secondary pipe branch or at the opposite side of the main branch pipe (Fig. 1). The wall temperature fluctuation can cause cyclical thermal stresses and subsequently fatigue cracking of the wall. The issue of thermal striping has been observed in light water reactors (LWRs) as several incidents of high-cycle fatigue at coolant mixing junctions have been detected - mainly in piping T-junctions - in nuclear plants, like the failure event at Civaux 1 , France. These incidents occurred usually in piping of diameter $5-20 \mathrm{~cm}$ and the most susceptible parts to thermal fatigue are mixing T-junctions of the Residual Heat Removal (RHR) system in both boiling (BWR) and pressurized water reactors (PWR). This has raised thermal fatigue to be a serious safety concern and an important aspect on ageing and life management of nuclear plants with LWR.

A typical value for the temperature difference between the hot/cold streams is $160^{\circ} \mathrm{C}$. Critical parameters for thermal fatigue analyses are frequencies $(\omega)$, temperature differences $(\Delta \mathrm{T})$, number of cycles $(\mathrm{N})$, and material properties. Most damaging thermal loads appear to be due to large scale turbulent fluctuations of low frequency (3-10 Hz). From a thermal hydraulic standpoint, the accurate prediction of such large coherent eddies is a challenging task, requiring $\mathrm{CFD}$ and advanced turbulence modelling.

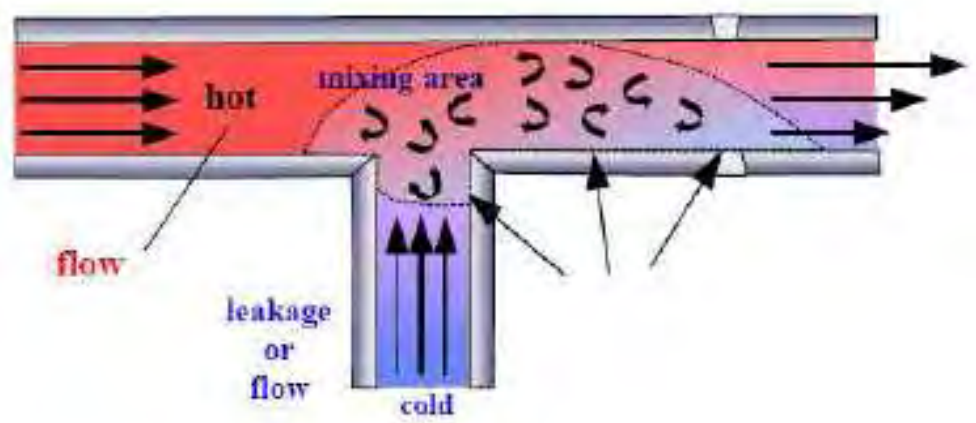

Significant effort has been put in the experimental investigation of the thermal fatigue and thermal

Fig. 1 T-junction mixing phenomena [7]

striping phenomena due to thermal mixing in pipe T-junctions. As example one can refer to the joint US-Japanese program, the program by EDF or the comprehensive, European Commission funded THERFAT project. Further experiments have been carried out by universities, vendors of nuclear power plant (NPP) equipment and NPP operators with the aim to 
provide detailed experimental data for the validation of Computational Fluid Dynamics (CFD) codes and model development.

In contrary to the turbulent mixing of two fluid streams of equal temperature, which can obviously quite accurately be described by traditional RANS models, the thermal mixing of two fluid streams of different temperature is a rather challenging case for computational fluid dynamics (CFD). The CFD methods based on RANS, which are typically used in industrial applications, have difficulties to provide accurate results for this flow situation. In many cases the high turbulent viscosity predicted from the RANS-based turbulence models in the mixing zone due to the locally high shear rates suppress any transient flow development and the CFD results tend to a steady-state solution. On the other hand experimental observations clearly state strong and high-frequency temperature transients at pipe walls downstream of the T-junction, the so-called thermal striping effect, which can lead to high-cycle thermal fatigue, crack formation and pipeline break in practical applications. Recent studies using advanced scale-resolving methods such as LES and DES have shown promising results (Braillard et al., [1]; Hu and Kazimi, [2]; Kuszaj and Komen, [3]; Ohtsuka et al., [4]; Westin et al., [5] and [6]). However, detailed validation of the tools and methods is still required in order to determine their range of validity and their expected accuracy.

In November 2008, a T-junction thermal mixing test was carried out at the Älvkarleby Laboratory of Vattenfall Research and Development (VRD) in Sweden. Data from this test have been reserved specifically for this CFD benchmark exercise [7].

\section{The Vattenfall T-Junction Experiment}

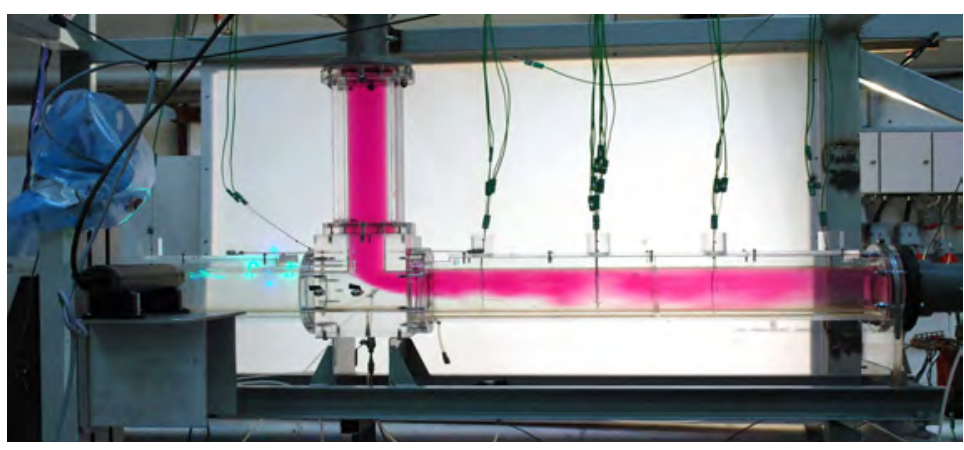

Fig. 2 Vattenfall T-Junction Experiment [7]
The test section is constructed from Plexiglas, and the junction itself from one solid block into which the main and branch pipes fit (Fig. 2). The dimensions are plotted in Table 1 . The temperatures of the water in the main and branch pipes were maintained at $15^{\circ} \mathrm{C}$ and $30^{\circ} \mathrm{C}$, respectively, with minimal heat loss. Special care was taken to provide simple and well-defined inlet boundary conditions to remove ambiguities in defining the CFD input data. Temperature fluctuations near pipe walls were measured using thermocouples. These were placed around the inner wall perimeter of the main pipe at seven stations downstream of the junction and at one station upstream (Fig. 3). All thermocouples were positioned $1 \mathrm{~mm}$ from the wall. Velocity profiles upstream and downstream of the junction were measured using a twocomponent LDV system. These were positioned at each inlet, and at the outlet. Data are in the form of mean values, RMS values and turbulence statistics.

The numerical prediction of thermal mixing and striping in terms of temperature amplitude and frequency using the current CFD technology is a computational intensive and challenging task. By the physics of the phenomenon, the flow is turbulent and highly transient and the thermal striping at pipe walls is affected by the formation and propagation of large-scale turbulent structures in space and time. The aim is therefore a CFD turbulence model validation study and a detailed CFD experiment comparison.

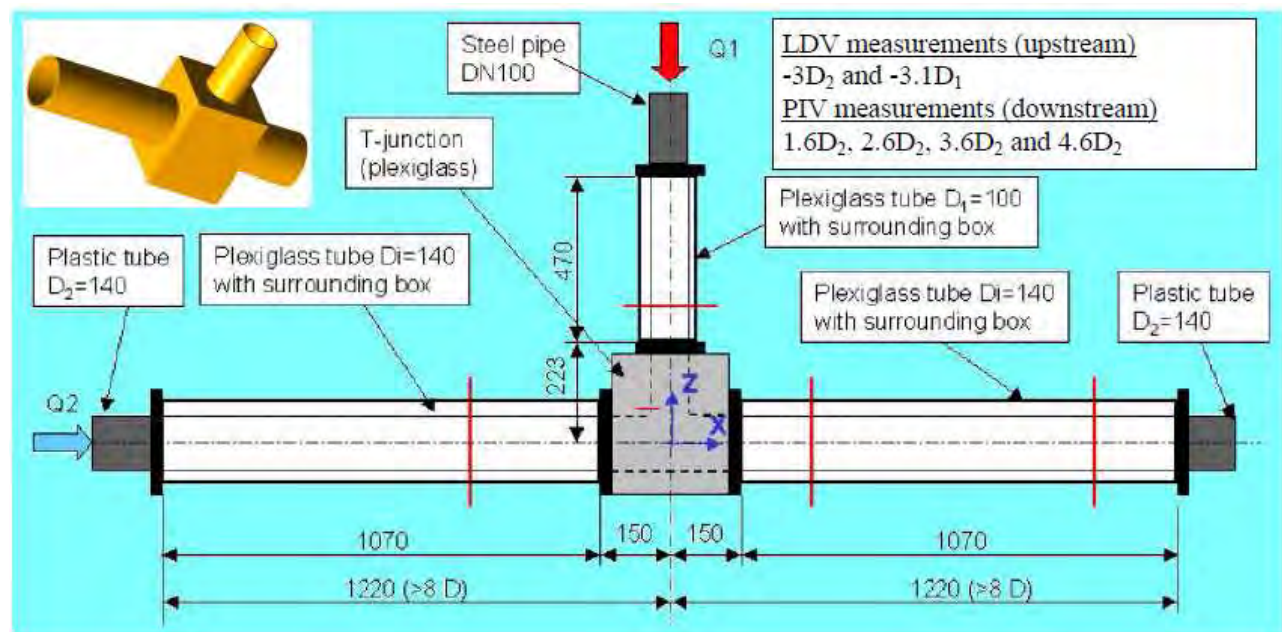

Fig. 3 Schematic of T-junction with Plexiglas sections [7] 
Turbulence model approaches to be studied in the present validation study include URANS SST as well as scale resolving turbulence models (LES).

Table 1: Dimensions of T-junction test section

\begin{tabular}{|l|l|l|}
\hline Component & Material & Dimensions (mm) \\
\hline Main inlet pipe & Plexiglas & Length: 1070; ID: 140; OD: 150 \\
\hline $\begin{array}{l}\text { Branch inlet } \\
\text { pipe }\end{array}$ & Plexiglas & Length: 470; ID: 100; OD: 110 \\
\hline Outlet pipe & Plexiglas & 1070; ID: 140; OD: 150 \\
\hline Tee Block & Plexiglas & $\begin{array}{l}\text { Length (x): 220; Width (y): 285, Height (z): } 325 \\
\text { Main channel diameter: 140 } \\
\text { Branch channel diameter: 100 }\end{array}$ \\
\hline
\end{tabular}

Vattenfall provided time averaged temperatures and temperature fluctuations for all thermocouples. They are located at $0^{\circ}$, $90^{\circ}, 180^{\circ}$, and $270^{\circ}$, two, four, six, eight, and ten hydraulic diameters downstream of the tee junction, and at $0^{\circ}$ and $180^{\circ}$, fifteen and twenty hydraulic diameters downstream of the tee junction (Fig. 4 and Fig. 6). In addition time dependent temperature readings were provided two and four hydraulic diameters downstream of the tee junction at the four angular locations, at $0^{\circ}, 180^{\circ}$, and $270^{\circ}$ six diameters downstream, and at $0^{\circ}, 90^{\circ}$, and $180^{\circ}$ eight diameters downstream. This data was collected every five milliseconds for three hundred seconds. Vattenfall also provided PIV data at 1.6, 2.6, 3.6, and 4.6 hydraulic diameters downstream of the tee junction (Fig., 4). Time averaged and RMS fluctuations were provided for the $\mathrm{x}$ and $\mathrm{z}$ velocity components ( $\mathrm{U}$ and $\mathrm{W}$ ) along a vertical line through the center of the pipe at the four $\mathrm{x}$ locations. Time averaged and RMS fluctuations were provided for the $\mathrm{x}$ and $\mathrm{y}$ velocity components ( $\mathrm{U}$ and $\mathrm{V}$ ) along a horizontal line through the center of the pipe at the same four $\mathrm{x}$ locations. Time dependent PIV information was provided at the same four downstream locations, but only for a restricted number of points. All three velocity components were provided on the pipe centerline. The $\mathrm{x}$ and $\mathrm{z}$ components $(\mathrm{U}$ and $\mathrm{W}$ ) were provided at $\mathrm{y}=0$ and $\mathrm{z}= \pm 35 \mathrm{~mm}$, and the $\mathrm{x}$ and $\mathrm{y}$ components provided at $\mathrm{z}=0$ and $\mathrm{y}= \pm 35 \mathrm{~mm}$. PIV data was sampled 60 times per second for 12 seconds, and two independent 12 second samples were provided for each velocity component at each location. The inlet velocity profiles presented in this section are taken from an earlier test [6] in which the volumetric flow ratio between the main and branch streams was 2 . In the present test (flow ratio 1.5), the flowrate in the hot inlet was kept the same as before $(6 \mathrm{l} / \mathrm{s})$, whereas the one in the cold inlet was $9.0 \mathrm{l} / \mathrm{s}$ instead of $12.0 \mathrm{l} / \mathrm{s}$ used previously. Since the velocity profile is fully developed in the cold inlet, the results presented below can simply be scaled to fit the present flow rate. A non-dimensional temperature also called mixing scalar $\Theta_{x, y, z}(t)$ is used at each thermocouple location. $\Theta_{x, y, z}(t)$ is the actual temperature minus the cold flow inlet temperature, divided by the difference between hot and cold inlet temperatures:

$$
\Theta_{x, y, z}(t)=\frac{T_{x, y, z}(t)-T_{0}}{T_{1}-T_{0}}
$$

\section{Turbulence Modeling}

The CFD calculation was performed using ANSYS CFX-12 [8].

\section{RANS SST}

The two equation turbulence model "Shear Stress Transport Model (SST)" was used in a steady state CFD calculation to prescribe the initial condition. The SST model works by solving a turbulence/frequency-based model $(\mathrm{k}-\omega)$ at the wall and standard $\mathrm{k}-\omega$ in the bulk flow. A blending function ensures a smooth transition between those two models (Menter, [9]).

The kinematic eddy viscosity is calculated according to Eq. (1):

$$
v_{T}=\frac{a_{1} k}{\max \left(a_{1} \omega, S F_{2}\right)}
$$

In the SST model the turbulence kinetic energy can be prescribed:

$$
\cdot \frac{\partial k}{\partial t}+U_{j} \frac{\partial k}{x_{j}}=P_{k}-\beta^{*} k \omega+\frac{\partial}{\partial x_{j}}\left[\left(v+\sigma_{k} v_{t}\right) \frac{\partial k}{\partial x_{j}}\right]
$$


Similar the Specific dissipation rate is formulated:

$$
\frac{\partial \omega}{\partial t}+U_{j} \frac{\partial \omega}{x_{j}}=\alpha S^{2}-\beta \omega^{2}+\frac{\partial}{\partial x_{j}}\left[\left(v+\sigma_{\omega} v_{T}\right) \frac{\partial \omega}{\partial x_{j}}\right]+2\left(1-F_{1}\right) \sigma_{\omega 2} \frac{1}{\omega} \frac{\partial k}{\partial x_{i}} \frac{\partial \omega}{\partial x_{i}}
$$

\section{Large Eddy Simulation (LES)}

LES turbulence model was used in the transient calculation. Large eddies of the turbulence are computed and only the smallest eddies are modeled. Usually, the computational grid serves as a low pass filter and only the subgrid scale turbulent phenomena are modeled. This feature allows one to explicitly solve for the large eddies in a calculation and implicitly account for the small eddies by using a subgrid-scale model (SGS model).

Subgrid-scale turbulence models usually employ the Boussinesq hypothesis, and seek to calculate (the deviatoric part of) the SGS stress using:

$$
\tau_{i j}-\frac{1}{3} \tau_{k k} \delta_{i j}=-2 \mu_{t} \bar{S}_{i j}
$$

where $\bar{S}_{i j}$ is the rate-of-strain tensor for the resolved scale defined by

$$
\bar{S}_{i j}=\frac{1}{2}\left(\frac{\partial \bar{u}_{i}}{\partial x_{j}}+\frac{\partial \bar{u}_{j}}{\partial x_{i}}\right)
$$

and $v_{t}$ is the subgrid-scale turbulent viscosity. Substituting into the filtered Navier-Stokes equations, we then have

$$
\frac{\partial \bar{u}_{i}}{\partial t}+\bar{u}_{j} \frac{\partial \bar{u}_{i}}{\partial x_{i}}=-\frac{1}{\rho} \frac{\partial \bar{p}}{\partial x_{i}}+\frac{\partial}{\partial x_{i}}\left(\left[v+v_{t}\right] \frac{\partial \bar{u}_{i}}{\partial x_{j}}\right)
$$

where we have used the incompressibility constraint to simplify the equation and the pressure is now modified to include the trace term $\tau_{k k} \delta_{i j} / 3$.

In the WALE model [10] the eddy viscosity is modeled by:

$$
\mu_{t}=\rho \Delta_{s}^{2} \frac{\left(S_{i j}^{d} S_{i j}^{d}\right)^{3 / 2}}{\left(\bar{S}_{i j} \bar{S}_{i j}\right)^{5 / 2}+\left(S_{i j}^{d} S_{i j}^{d}\right)^{5 / 4}} \quad \text { with } \Delta_{s}=C_{\omega} V^{1 / 3}
$$

where $\mathrm{S}$ denotes the traceless symmetric part of the square of the velocity gradient tensor:

$$
S_{i j}^{d}=\frac{1}{2}\left(\bar{g}_{i j}^{2}+\bar{g}_{j i}^{2}\right)-\frac{1}{3} \delta_{i j} \bar{g}_{k k}^{2}
$$

where $\bar{g}_{i j}=\frac{\partial \bar{u}_{i}}{\partial x_{j}}$ and $\mathrm{d}_{\mathrm{ij}}$ is the Kronecker symbol.

The main advantages of the WALE model are the capability to reproduce the laminar to turbulent transition and the design of the model to return the correct wall-asymptotic -variation of the SGS viscosity. It offers therefore the same advantage as 
the Dynamic Smagorinsky model and at the same time does not require an explicit filtering. The constant $C_{W}$ has been calibrated using freely decaying isotropic homogeneous turbulence: the default value is 0.5 .

\section{CFD simulation setup and boundary conditions}

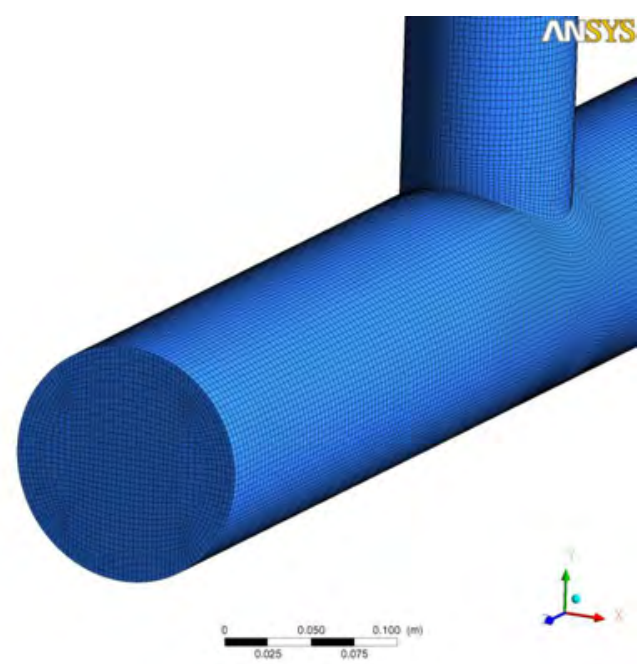

Fig. 4 Grid model

A hexahedral mesh was generated with about 1 Mio. nodes (Fig. 4). The averaged $\mathrm{y}^{+}$value at the pipe wall was 26 . The mesh was generated scalable with minimum mesh angles of about $40^{\circ}$. For the CFD investigation the inlet geometry was shortened to the locations of the LDV measurements in the cross-sections in the main and branch pipe in front of the T-junction (see Fig. 4), since exactly the measured mean velocity profiles from the experimental data were prescribed here as inlet boundary conditions for the CFD simulation. Therefore inlet boundary conditions (see Table 2) have been prescribed at the upstream cross sections at $\mathrm{z}$ $=-3 \mathrm{D} 2$ for the cold leg (main pipe) and at $\mathrm{y}=-3.1 \mathrm{D} 1$ for the hot leg (branch pipe). Profiles of turbulent kinetic energy and turbulent dissipation were derived from the LDV data for both inlets. No transient inflow boundary conditions, e.g. generation of unsteady velocity fluctuations according to turbulence spectra or use of unsteady pipe flow data, has been prescribed at the inlets. A zero averaged static pressure outlet $\mathrm{BC}$ has been used for the outlet cross-section and non-slip BC's with automatic wall treatment are used for all walls of the domain. Additionally so-called monitoring points were introduced at all locations of thermocouples, in order to monitor the transient history of main characteristics, i.e. fluid temperature at these locations for comparison with the data. Some basic investigations have been carried out for the verification of the CFD setup according to the Best Practice Guidelines BPG [11]. The temperature dependent fluid properties (density, viscosity) have been taken into account by defining the water properties from the industry standard IAPWS-IF97. For the transient LES simulation a second order backward Euler time discretization with a time step of $\mathrm{t}=$ $0.001 \mathrm{~s}$ was used and a convergence criterion based on the maximum residuals of $10^{-4}$ was reached at every time step with 3-5 coefficient loops (sub-iterations) per times. The central difference advection scheme has been applied for the spatial discretization of momentum equations. It was found, that after some initial transient behavior the RANS SST solution was quickly approaching a steady state solution in terms of velocity and temperature fields. This result underlines the strong requirement for scale-resolving turbulence models for this type of applications. The transient LES calculation took several weeks.

Table 2: Inlet temperatures and flow rates

\begin{tabular}{|l|l|l|l|l|}
\hline Inlet/designation & $\begin{array}{l}\text { Temperature } \\
\left({ }^{\circ} \mathbf{C}\right)\end{array}$ & $\begin{array}{l}\text { Pipe diameter } \\
(\mathbf{m m})\end{array}$ & $\begin{array}{l}\text { Measuring } \\
\text { location } \\
(\mathbf{m m}) \#\end{array}$ & $\begin{array}{l}\text { Volumetric flow } \\
\text { rate } \\
(\text { litres/s })\end{array}$ \\
\hline Main/InCo & 19 & $140\left(\mathrm{D}_{2}\right)$ & $-420\left(-3 \mathrm{D}_{2}\right)$ & 9.0 \\
\hline Branch/InHo & 36 & $100\left(\mathrm{D}_{1}\right)$ & $-310\left(-3.1 \mathrm{D}_{1}\right)$ & 6.0 \\
\hline
\end{tabular}

\section{Results}

\section{Qualitative Analysis}

As already mentioned, the LES Wale solution for the Vattenfall test case geometry was initialized at $\mathrm{t}=0.0 \mathrm{~s}$ with the steady-state result from the preceding SST RANS simulation. Then the transient simulation by using the LES scaleresolving turbulence model approach has been carried out for $19.5 \mathrm{~s}$ real time with a time step of $\Delta \mathrm{t}=0.001 \mathrm{~s}$, where after a first $5 \mathrm{~s}$ the transient averaging of mean flow field characteristics (e.g. mean velocity and temperature) has been started. Fig. 5 shows the typical developing vortex structures downstream of the $\mathrm{T}$-junction at $\mathrm{T}=15.5 \mathrm{~s}$ real time.

$$
Q=\Omega^{2}-S^{2}=\left(\frac{\partial u_{i}}{\partial x_{j}}-\frac{\partial u_{j}}{\partial x_{i}}\right)^{2}-\left(\frac{\partial u_{i}}{\partial x_{j}}+\frac{\partial u_{j}}{\partial x_{i}}\right)^{2}
$$


The visualization is based on isosurfaces of the so-called Q-criteria, where $\Omega$ being the vorticity and $\mathrm{S}$ the strain rate of the flow field (Eq. 10). The figure clearly shows that the small change in the Vattenfall T-junction geometry leads to the formation of a so-called horseshoe vortex upstream the intruding hot water jet from the branch pipe. Furthermore the figure shows rather irregular turbulent vortex structures forming immediately downstream of the T-junction and being convected with the flow along the main pipe. Further downstream it can be observed, that the length scale of the vortex structures decreases with turbulent dissipation. Fig. 6 shows the time averaged temperature distribution in cross-section at $\mathrm{z}=6 \mathrm{D}$ and $\mathrm{x}=0$. In this cross-section the enhanced turbulent mixing has led to a propagation of high temperature liquid along the upper pipe walls, where rather high averaged fluid temperatures can now be observed at the $\varphi=135^{\circ}$ and $\varphi=225^{\circ}$ thermocouple locations.

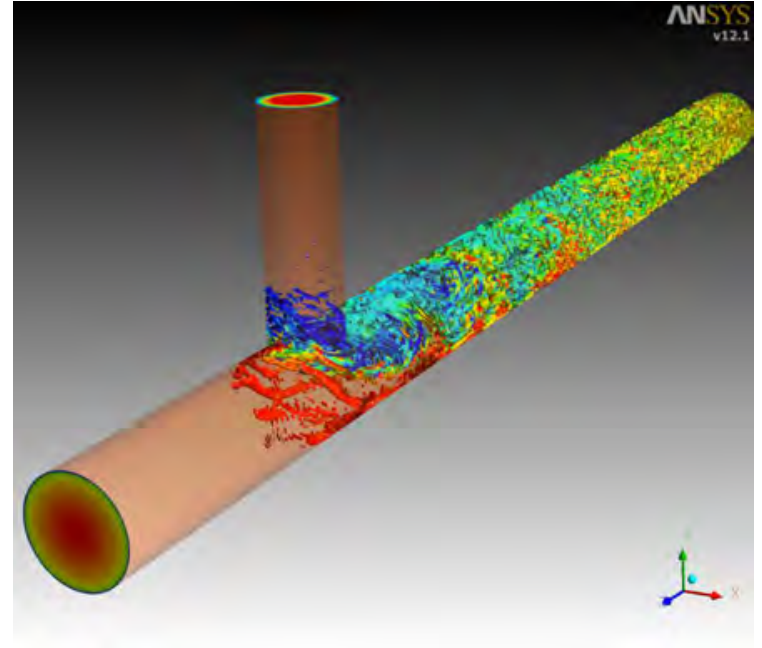

Fig. 5 Vortex structure Q criteria plotted with the Mixing Scalar at $19.9 \mathrm{~s}$

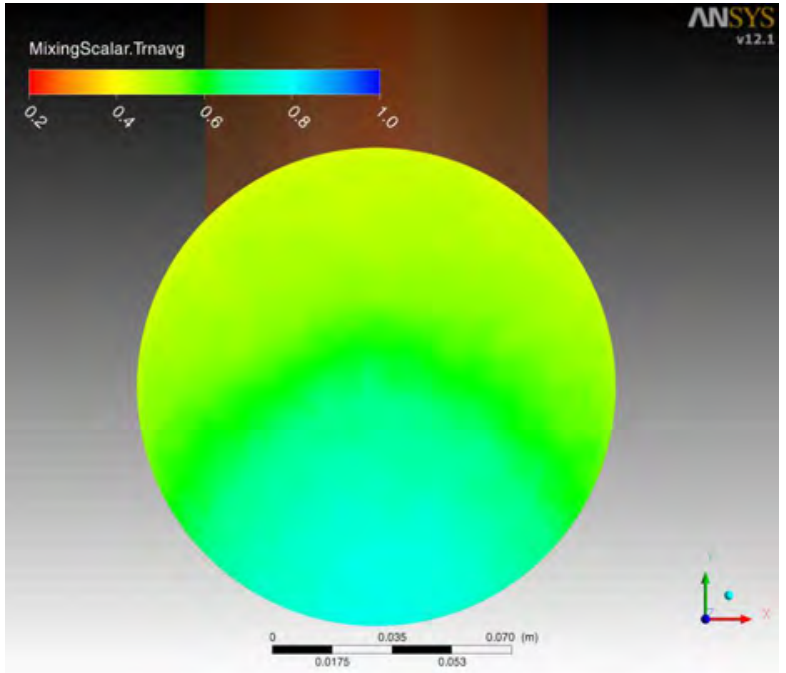

Fig. 6 Averaged value of the Mixing Scalar at 6D plane

\section{Quantitative Comparison}

Fig. 7 shows the averaged tangential velocity and Fig. 8 the averaged streamwise velocity at $4.6 \mathrm{D}$ in direct comparison to the LDV measurement data at these locations. In both Figs. 7 and 8 the comparison of LES simulation results indicate, that scale-resolving CFD simulation shows very good agreement with averaged experimental data. The time series of recorded thermocouple measurements in comparison to the fluid temperature recorded at the corresponding monitoring point locations show similar patterns. Fig. 9 shows the comparison of transient temperature signals for the thermocouples $8 \mathrm{D}$ at $90^{\circ}$ position. In both the CFD results and experimental data no regular frequency of the temperature fluctuation over time can be identified. The CFD results show in comparison to experiments similar amplitudes of temperature fluctuations. In Fig. 10 the corresponding velocity components at this local position are plotted.

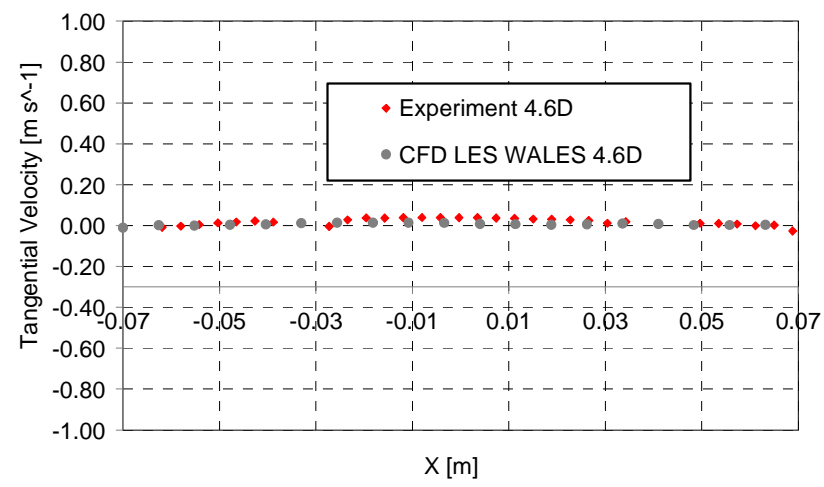

Fig. 7 Averaged tangential velocity at $4.6 \mathrm{D}$

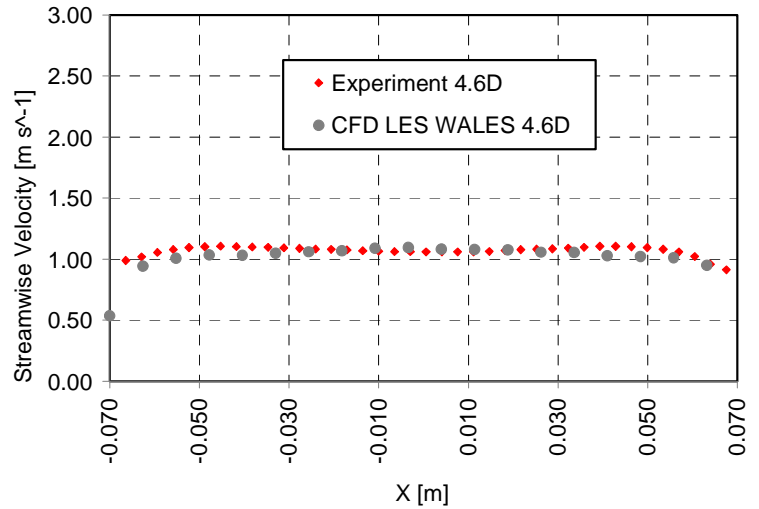

Fig. 8 Averaged streamwise velocity at $4.6 \mathrm{D}$ 


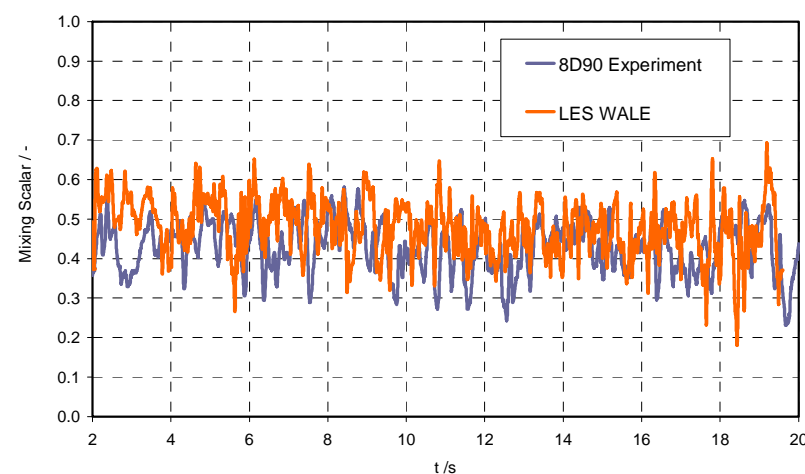

Fig. 9 Local fluctuations of Mixing Scalar at 8D 90

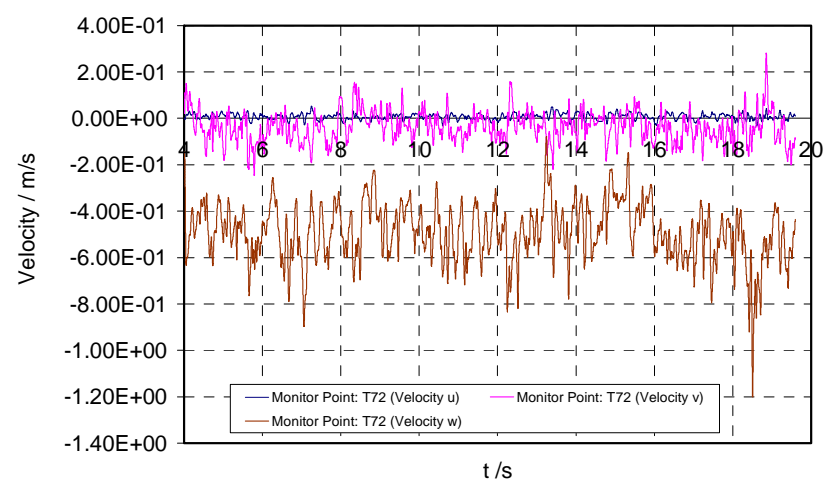

Fig. 10 Local fluctuations of velocity components at 8D $90^{\circ}$

\section{Fourier Analysis}

The primary goal of Fourier analysis is to check for simulation of periodic low frequency flow oscillations. A secondary goal is to compare the turbulence spectrum predicted by LES with that seen in data. At data locations (e.g. thermocouple 4D270) showed spectral peaks in both CFD and experimental data near 3-5 Hz (Figs. 11 and 12). Fig. 11 indicates, that more statistical date are needed for the CFD simulation to cover a large frequency spectrum from $10^{-3}$ to $1 \mathrm{~Hz}$.

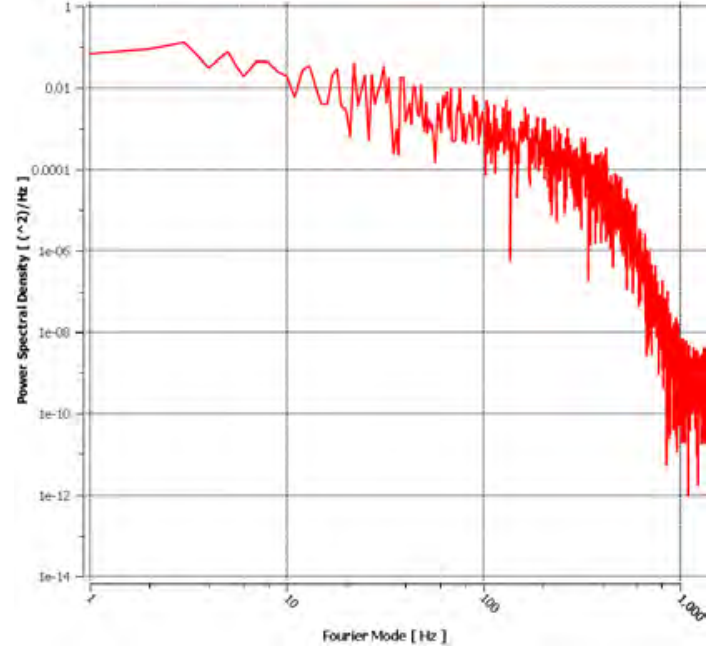

Fig. 11 Power spectral density of temperature fluctuation over frequency 4D270 (LES Wale)

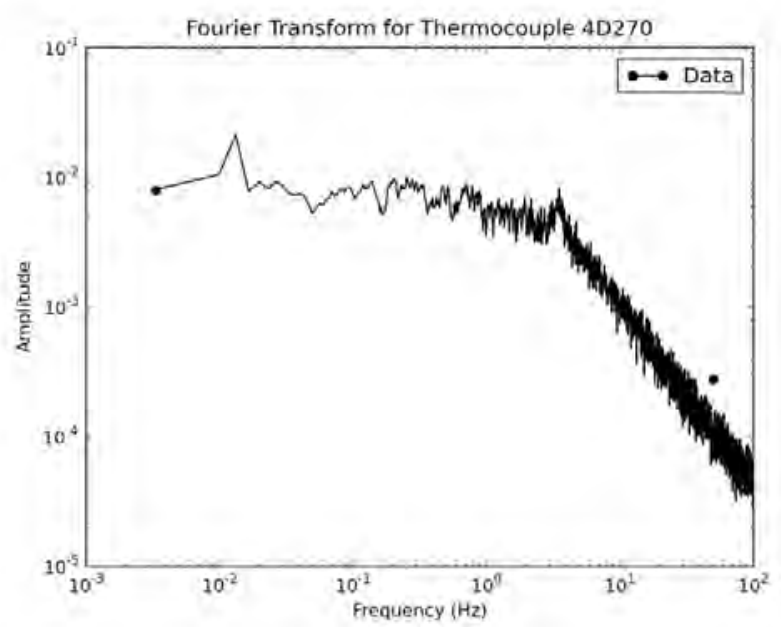

Fig. 12 Power spectral density of temperature fluctuation over frequency 4D270 (Thermocouple Experiment, [7]

\section{Summary}

Thermal mixing of water streams of different temperature in T-junctions is a challenging test case for CFD methods, and advanced scale-resolving turbulence modeling approaches like LES are required in order to simulate the strongly transient flow and temperature fields. The application of transient LES simulations and the validation of the scale-resolving turbulence models today still involves a couple of unresolved questions due to the extremely high computational effort in the order of several weeks for a single transient CFD simulation.

The investigated test case was provided by Vattenfall AB Sweden in the frame of a OECD/NEA T-junction benchmark and is aimed to provide detailed experimental validation data and thoroughly prepared and monitored test case boundary conditions for the assessment and validation of LES turbulence models for the further study of thermal striping phenomena occurring in thermal mixing in T-junctions. ANSYS CFX 12.0 with the LES Wale approach has been applied to the provided testcase conditions. The CFD solution showed the occurrence of the thermal striping phenomena in the simulations. Predicted general flow patterns and time averaged mean velocity profiles are in good agreement with the experimental observations. Spectral peaks in both CFD and experimental data were found near 3-5 Hz. Further 
investigations are carried out in order to investigate the influence of mesh resolution on statistically averaged flow simulation results.

\section{Acknowledgement}

The author like to acknowledge Kristian Angele from Vattenfall AB Sweden, who has provided the underlying detailed and very carefully documented sets of validation data.

\section{Literature}

[1] Braillard, O., Jarny, Y., Balmigere, G., 2005. Thermal load determination in the mixing Tee impacted by a turbulent flow generated by two fluids at large gap of temperature, ICONE13-50361. In: 13th International Conference on Nuclear Engineering, Beijing, China, May 16-20.

[2] Hu, L.-W., Kazimi, M.S., 2003. Large Eddy simulation of water coolant thermal striping in amixing Tee junction. In: The 10th Int. Topical Meeting in Nuclear Reactor Thermal Hydraulics (NURETH-10), Seoul, Korea, October 5-9, pp. 1-10.

[3] Kuszaj, A.K., Komen, E., 2008. Large-eddy simulations for thermal fatigue -An assessment on the flow characteristics. In: Presentation on the Meeting of the German CFD Network in Nuclear Reactor Safety, Grosshartpenning, Germany, 23-24 January, 29 pp.

[4] Ohtsuka, M., Kawamura, T., Fukuda, T., Moriya, S., Shiina, K., Kurosaki, M., Minami, Y., Madarame, H., 2003. LES analysis of fluid temperature fluctuations in a mixing Tee pipe with the same diameters. In: ICONE 11-36064, 11th International Conference on Nuclear Engineering, Tokyo, Japan, April 20-23

[5] Westin, J., Alavyoon, F., Andersson, L., Veber, P., Henriksson, M., Andersson, C., 2006. Experiments and unsteady CFD-calculations of thermal mixing in a T-junction. OECD/NEA/IAEA Workshop on the Benchmarking of CFD Codes for Application to Nuclear Reactor Safety (CFD4NRS), Munich, Germany, pp. 1-15.

[6] Westin, J., Veber, P., Andersson, L., 't Mannetje, C., Andersson, U., Eriksson, J., Hendriksson, M., Alavyoon, F., Andersson, C., 2008. High-cycle thermal fatigue in mixing Tees. Large-Eddy simulations compared to a new validation experiment. 16th Int. Conf. On Nuclear Engineering (ICONE-16), Florida, Orlando, USA, 11-15 May, pp. 1-11, Paper No. 48731.

[7] Brian Smith et all, OECD/NEA-VATTENFALL T-junction benchmark specifications, OECD/NEA Report, Juli 2009

[8] ANSYS CFX-13.0 User Manual, ANSYS Inc., Canonsburg, USA, November 2010.

[9] Menter, F. R. (1993), "Zonal Two Equation k- $\omega$ Turbulence Models for Aerodynamic Flows", AIAA Paper 932906.

[10] Nicoud, F. and Ducros, F. (1999), "Subgrid-scale modelling based on the square of the velocity gradient tensor", Flow, Turbulence and Combustion, 62: pp- 183-200, 1999

[11] Menter, F.R., 2002. CFD best practice guidelines for CFD code validation for reactor safety applications, EC Project ECORA, Report EVOL-ECORA-D01, pp. 1-47. 